\title{
A DETAILED HISTOLOGIC ANALYSIS OF PULMONARY ARTERIOVENOUS MALFORMATIONS IN CHILDREN WITH CYANOTIC CONGENITAL HEART DISEASE
}

Brian W. Duncan, MD

James M. Kneebone, MS ${ }^{\mathrm{a}}$

Emil Y. Chi, MD ${ }^{\mathrm{b}}$

Viktor Hraska, MD

F. Frank Isik, MD ${ }^{d}$

Geoffrey L. Rosenthal, MD, $\mathrm{PhD}^{\mathrm{e}}$

Thomas K. Jones, MD

Sandra L. Starnes, MD ${ }^{a}$

Flavian M. Lupinetti, MD
Introduction: Pulmonary arteriovenous malformations are a common cause of progressive cyanosis in children after cavopulmonary anastomoses. We analyzed the pulmonary histologic characteristics from children in whom pulmonary arteriovenous malformations developed after procedures that resulted in pulmonary arterial blood flow devoid of hepatic venous effluent. Methods: We performed routine histologic studies, immunohistochemical staining, and electron microscopic analysis of peripheral lung biopsy specimens from 2 children with angiographically proven pulmonary arteriovenous malformations. Microvessel density was determined with a computer-assisted, morphometric analysis system. Results: Histologic examination demonstrated large, dilated blood vessels ("lakes") and clustered, smaller vessels ("chains") in the pulmonary parenchyma. Microvessel density was significantly greater in these patients than in age-matched controls $(P=.01)$. Immunohistochemistry demonstrated uniform staining for type IV collagen and $\alpha$ smooth muscle actin, weak staining for the endothelial marker CD31 (cluster of differentiation, PECAM-1), and negative staining for proliferating cell nuclear antigen. Electron microscopy revealed endothelial irregularity, a disorganized basement membrane, and increased numbers of collagen and actin filaments beneath the endothelium. Conclusions: This study represents an attempt to characterize the histologic features of pulmonary arteriovenous malformations in children with congenital heart disease who have pulmonary arterial blood flow devoid of hepatic venous effluent. The histologic correlate of this condition appears to be greatly increased numbers of thin-walled vessels. Immunohistochemistry suggests that the rate of cellular proliferation is not increased in these lesions. The development of these techniques may provide a standardized histologic approach for this condition and aid in understanding its etiology. (J Thorac Cardiovasc Surg 1999;117:931-8)
$D$ ulmonary arteriovenous (AV) malformations were first reported as a cause of progressive cyanosis in children after a classic Glenn anastomosis. ${ }^{1}$ Subsequent

From the Divisions of Cardiac Surgery a and Cardiology, ${ }^{\mathrm{e}}$ Children's Hospital and Regional Medical Center, Seattle; the Departments of Pathology ${ }^{\mathrm{b}}$ and Surgery, ${ }^{\mathrm{d}}$ University of Washington, Seattle, Wash; and the Department of Cardiovascular Surgery, ${ }^{c}$ The Children's Hospital, Bratislava, Slovakia.

Received for publication Aug 17, 1998; revisions requested Oct 30, 1998; revisions received Dec 29, 1998; accepted for publication Jan 13, 1999.

Address for reprints: Brian W. Duncan, MD, Division of Cardiac Surgery, Children's Hospital and Regional Medical Center, 4800 Sand Point Way, NE, PO Box 5371/CM-03, Seattle, WA 98105.

Copyright (C) 1999 by Mosby, Inc.

$0022-5223 / 99 \$ 8.00+0 \quad \mathbf{1 2 / 1 / 9 7 0 5 5}$ reports have identified several groups of children with congenital heart disease who are at risk for the development of pulmonary AV malformations. ${ }^{2,3}$ All of these patients share the absence of hepatic venous effluent directly perfusing the pulmonary circulation, usually after a cavopulmonary anastomosis. Although the development of pulmonary AV malformations in these children has been a clinically recognized phenomenon for more than 30 years, little is known about their exact nature or their etiology. We have previously approached the development of pulmonary AV malformations in children after cavopulmonary anastomosis as a form of abnormal angiogenesis under hepatic control. ${ }^{4}$ The present study gives a detailed pathologic description of the pulmonary parenchyma in 2 children in whom pulmonary AV malformations developed after procedures 


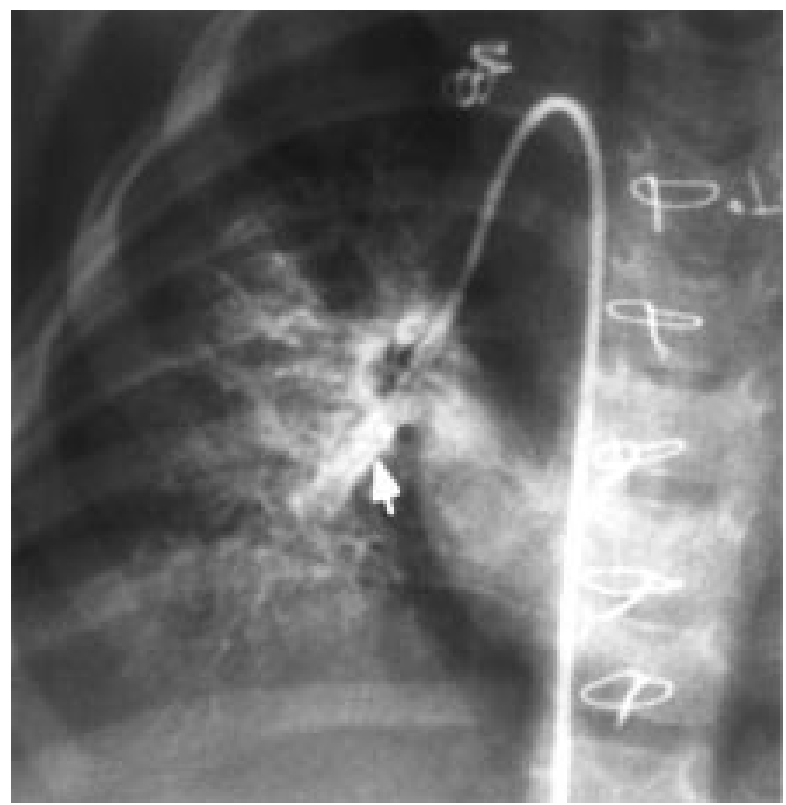

Fig 1. Selective injection of the right lower lobe pulmonary artery of the first patient, demonstrating diffuse pulmonary AV malformations. Rapid filling of the pulmonary vein (arrow) is seen in the capillary phase of injection.

that resulted in pulmonary arterial blood flow devoid of hepatic venous effluent.

\section{Methods \\ Clinical summaries}

PATIENT 1. A girl, aged 2 years 10 months, had single ventricle physiology consisting of double-outlet right ventricle, pulmonary atresia, bilateral superior venae cavae (SVCs), and interrupted inferior vena cava (IVC) with azygos continuation to the right SVC. The patient had undergone a left bidirectional Glenn shunt as her initial palliative procedure at 7 months of age. She subsequently underwent a Kawashima procedure with anastomosis of the right-sided SVC to the right pulmonary artery. After the procedure the patient's initial transcutaneous oximetric examination while breathing room air demonstrated oxygen saturations of $80 \%$. However, progressive cyanosis subsequently developed, with saturations consistently in the 50\% to $60 \%$ range. Angiography revealed diffuse pulmonary AV malformations (Fig 1). The patient underwent baffling of the hepatic veins to the right pulmonary artery via a fenestrated extracardiac pericardial conduit. She has subsequently had substantial improvement in her transcutaneous oxygen saturation, which is currently $83 \%$. Residual right-to-left shunting persists across the baffle fenestration.

PATIENT 2. A 12-year-old boy had visceral heterotaxia (polysplenia). Other abnormalities included dextrocardia with a large ventricular septal defect, right ventricular out- flow tract obstruction, secundum atrial septal defect, left SVC, interrupted IVC with azygos continuation, and drainage of the hepatic veins to the left atrium. The patient had undergone closure of the ventricular septal defect, resection of the right ventricular outflow obstruction, and closure of the atrial septal defect at 5 years of age. The hepatic veins were left draining to the left atrium, with systemic saturations of $95 \%$ initially after the operation. Over the next 5 years, progressive cyanosis developed, with room air saturations of $60 \%$ and angiographically confirmed pulmonary $\mathrm{AV}$ malformations. The patient underwent creation of an intracardiac tunnel to direct the hepatic veins to the right atrium and currently has oxygen saturations of approximately $95 \%$.

Histology and microvessel density. Lung biopsy specimens were obtained during the most recent operation in both patients to aid in determining the cause of progressive hypoxemia. Surgical consent was obtained in each case. A single biopsy specimen was obtained from the periphery of the lingula in each patient without an effort to obtain tissue from the regions of the lung that were most severely affected according to the angiograms. All studies were performed on the surgical specimens from these patients. Lung tissue was also examined from age-matched controls, archived specimens obtained from children who did not have cardiac disease. One specimen came from an autopsy of a child who died of viral encephalomyelitis. The other specimen was from a peripheral lung biopsy performed in a child with mild pulmonary insufficiency; the tissue demonstrated no diagnostic abnormality. Lung biopsy tissue had been fixed in Methacarn (60\% methanol, $30 \%$ chloroform, $10 \%$ acetic acid) or $10 \%$ neutral buffered formalin, dehydrated in graded alcohols, xylene infiltrated, and embedded in paraffin. Serial sections were cut $5-\mu \mathrm{m}$ thick, cleaned of paraffin, and rehydrated in xylenes and graded alcohols. Sections were stained for routine histologic evaluation with hematoxylin and eosin or Movat's pentachrome. Microvessel density was determined by counting the number of blood vessels in 10 randomly selected highpower fields $(200 \times)$ in histologic sections stained with Movat's pentachrome by means of a computerized system ${ }^{5}$ (Bioscan Optimas Image Analysis, Bothell, Wash) and expressed as the number of blood vessels per high-power field. This analysis was performed and the results were compared between the patients having pulmonary AV malformations and the archived age-matched controls.

Immunohistochemistry. Histologic sections were pretreated as necessary with protease $(0.01 \%$ weight/volume $)$ and sodium citrate $(10 \mu \mathrm{mol} / \mathrm{L})$, and endogenous peroxidase was quenched with hydrogen peroxide (3\% volume/volume). Nonspecific binding sites were blocked with nonfat dry milk (5\% weight/volume) and/or normal horse serum (50 $\mu \mathrm{L} / 10$ $\mathrm{mL}$ ) before addition of primary antibodies. Primary antibodies used were as follows: proliferating cell nuclear antigen (PCNA), CD31 (cluster of differentiation, PECAM-1), collagen type IV (all from DAKO, Carpinteria, Calif), and $\alpha$ smooth muscle actin (Boehringer-Manheim, Indianapolis, Ind). Primary antibodies were diluted in phosphate-buffered saline solution and applied to the sections for 1 hour. 


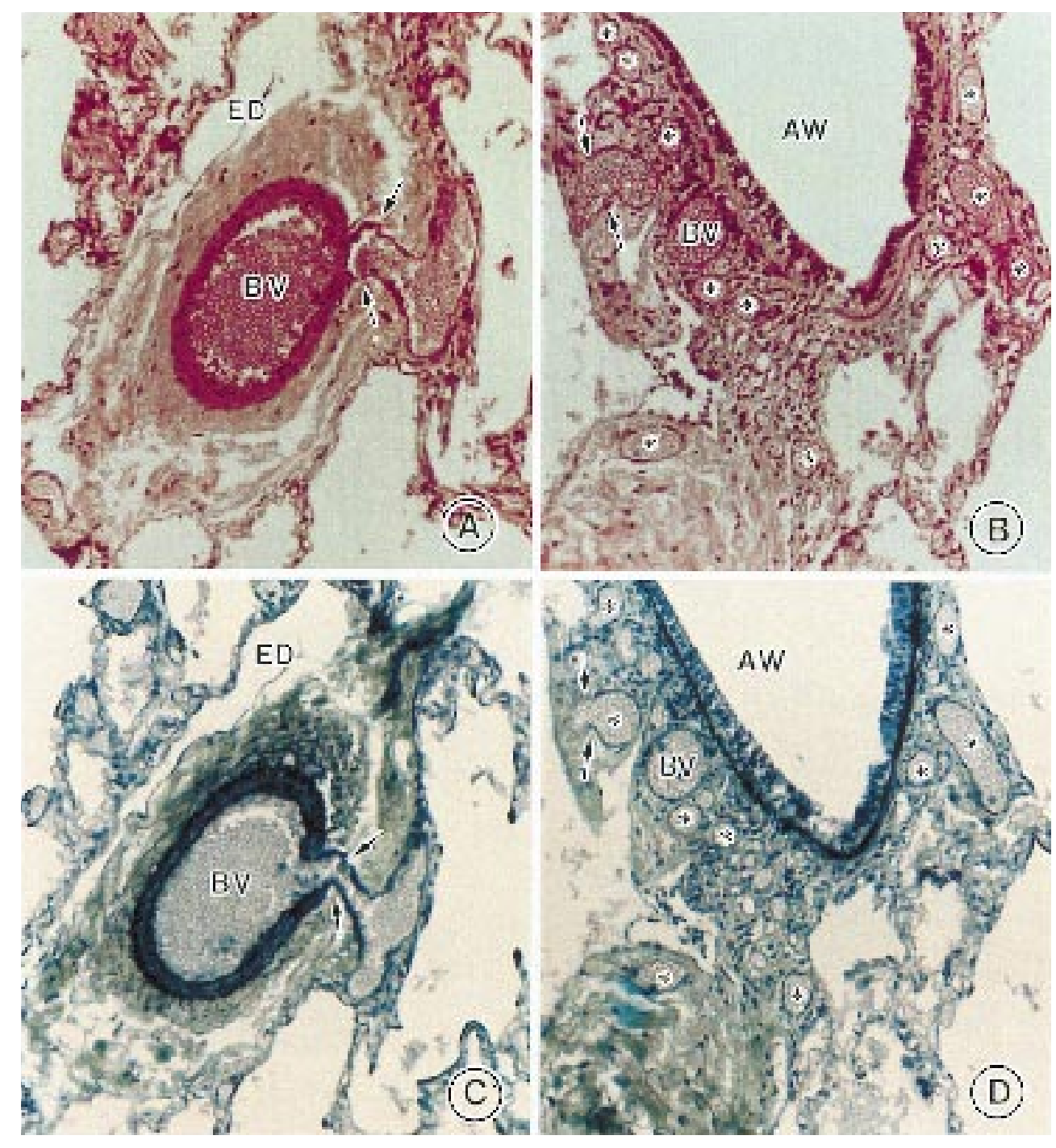

Fig 2. Micrographs of a lung biopsy specimen from the first patient with pulmonary AV malformations. A, Slight hypertrophy of the muscle of pulmonary arteriole $(B V)$ with distended, irregular, thin-walled vessel arising from arteriole (arrows ) ("lake" type of pulmonary AV malformations). Edema ( $E D$ ) of the pulmonary parenchyma is evident in a perivascular location. (Hematoxylin and eosin stain, 200×). B, Airway $(A W)$ surrounded by numerous, small, thin-walled blood vessels ( $B V$ and *) ("chain" type of pulmonary AV malformations). Cross-sectioned vessels demonstrate their irregular shape (arrows). (Hematoxylin and eosin, 200×.) C, Origin of pulmonary AV malformation "lake" branching from pulmonary arteriole $(B V)$ stained for elastin, demonstrating fibrous and elastin intimal thinning of pulmonary AV malformations (arrows). (Movat's pentachrome, 200×.) D, Pulmonary AV malformation "chains" ( $B V$ and $*)$ surrounding an airway $(A W)$ with thin walls demonstrating incomplete elastin staining (arrows) (Movat's pentachrome, 200×.)

Secondary antibodies (horse antimouse or horse antirabbit [both from DAKO] depending on the primary antibody) were diluted 1:500, applied for 30 minutes, and washed in phosphate-buffered saline solution. Avidin-biotin complex (Vector, Burlingame, Calif) was applied for 30 minutes, washed in phosphate-buffered saline solution, and pretreated with Trisma:HCl buffer (Sigma Chemical Co, St Louis, Mo).
Slides were then immersed in chromagen complex (diaminobenzidine, $5 \mu \mathrm{g}), 3 \%$ hydrogen peroxide $(750 \mathrm{~mL})$, and Trisma: $\mathrm{HCl}$ buffer $(175 \mathrm{~mL})$ and counterstained with methyl green. Appropriate positive and negative controls were examined to determine that staining was specific.

Electron microscopy. Tissue was fixed for 2 hours at $4{ }^{\circ} \mathrm{C}$ in Karnovsky's fixative (4\% formaldehyde and 5\% glu- 


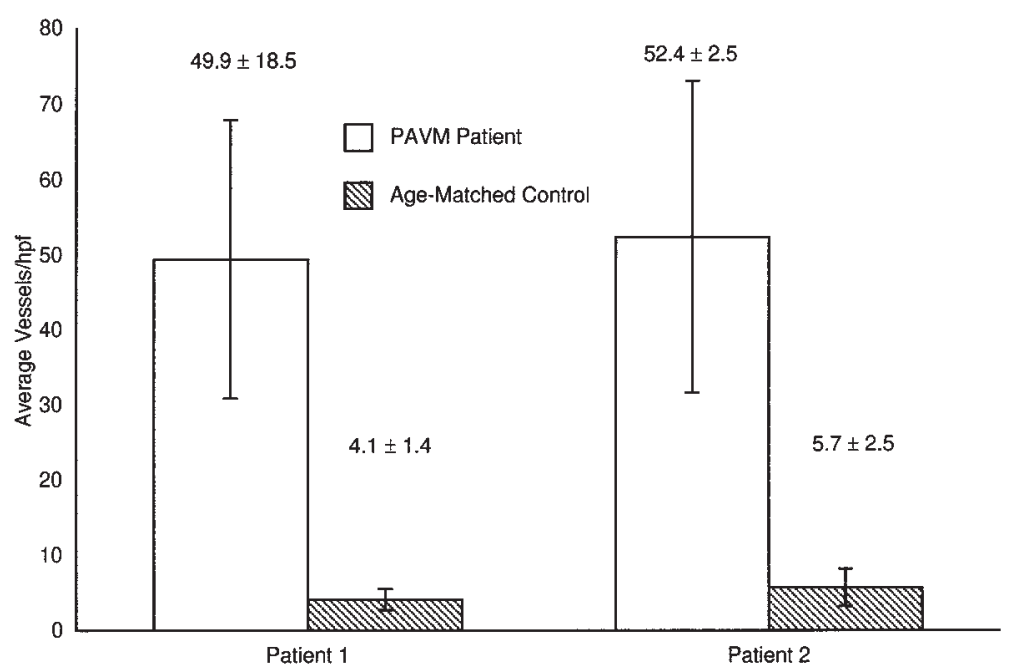

Fig 3. Microvessel densities of patients with pulmonary AV malformations (PAVM) versus age-matched controls. Microvessel density was determined for 10 high-power $(200 \times)$ fields from histologic sections stained with Movat's pentachrome for both the patients with pulmonary AV malformations and the age-matched controls and are expressed as the mean \pm the standard deviation.

taraldehyde), postfixed in $2 \%$ osmium tetroxide, and embedded in Epon fixative. Sections $1-\mu \mathrm{m}$ thick were stained with toluidine blue. Thin sections were prepared and stained with uranyl acetate and lead citrate. These sections were photographed with a JEOL 100B transmission electron microscope (JEOL USA, Peabody, Mass).

Statistical analysis. The average microvessel density for the 10 high-power microscopic fields was determined for both patients and their age-matched controls. The mean of the differences of the average microvessel density for the patients with pulmonary AV malformations and their age-matched controls was compared by means of the paired $t$ test.

\section{Results}

In comparison with specimens from age-matched controls, lung tissue from the patients having pulmonary AV malformations demonstrated greatly increased numbers of vessels extending to the periphery of the lung. The vessels were thin walled, with deficient elastin staining by Movat's pentachrome. Some of these appeared as "lakes" of large, dilated blood vessels whereas others demonstrated "chains" of clustered, smaller blood vessels. Some sections demonstrated branching of thin-walled vessels directly from precapillary arterioles (Fig 2).

The microvessel density from the specimens with pulmonary AV malformations was significantly greater than in age-matched controls. The mean \pm standard deviation of the differences in the average microvessel density between the 2 patients with pulmonary AV malforma- tions and their age-matched controls was $46 \pm 0.10$ vessels per high-power field (confidence interval, 44.6-47.4; $P=.01)$. Fig 3 demonstrates the mean microvessel density \pm standard deviation for each patient with pulmonary AV malformations compared with their agematched controls for illustrative purposes.

PCNA staining was not increased in the patients with pulmonary AV malformations (Fig 4, $A$ and $B$ ). This suggested that either the pulmonary AV malformations were now stable in growth or that vessel dilation had occurred, which did not involve significant cellular proliferation. CD31 staining was generally diminished and nonuniform throughout the pulmonary AV malformations, with some vessels demonstrating light staining and other vessels not staining at all (Fig 4, $C$ and $D$ ). There was no difference in the CD31 staining pattern for vessels of chain or lake morphology. Basement membrane staining (type IV collagen) was evident in all pulmonary $\mathrm{AV}$ malformations, as was positive staining for $\alpha$-smooth muscle actin in most pulmonary AV malformations, including small chain-like vessels (not shown).

To define the vessel wall ultrastructure of the pulmonary AV malformations, we performed transmission electron microscopy, which revealed numerous areas of endothelial irregularity and thickening, with abundant "stress" fibers in the endothelial cytoplasm (Fig 5). The basement membrane was highly disorganized in these areas and had numerous collagen fibers. These findings of endothelial discontinuity are consistent with the 


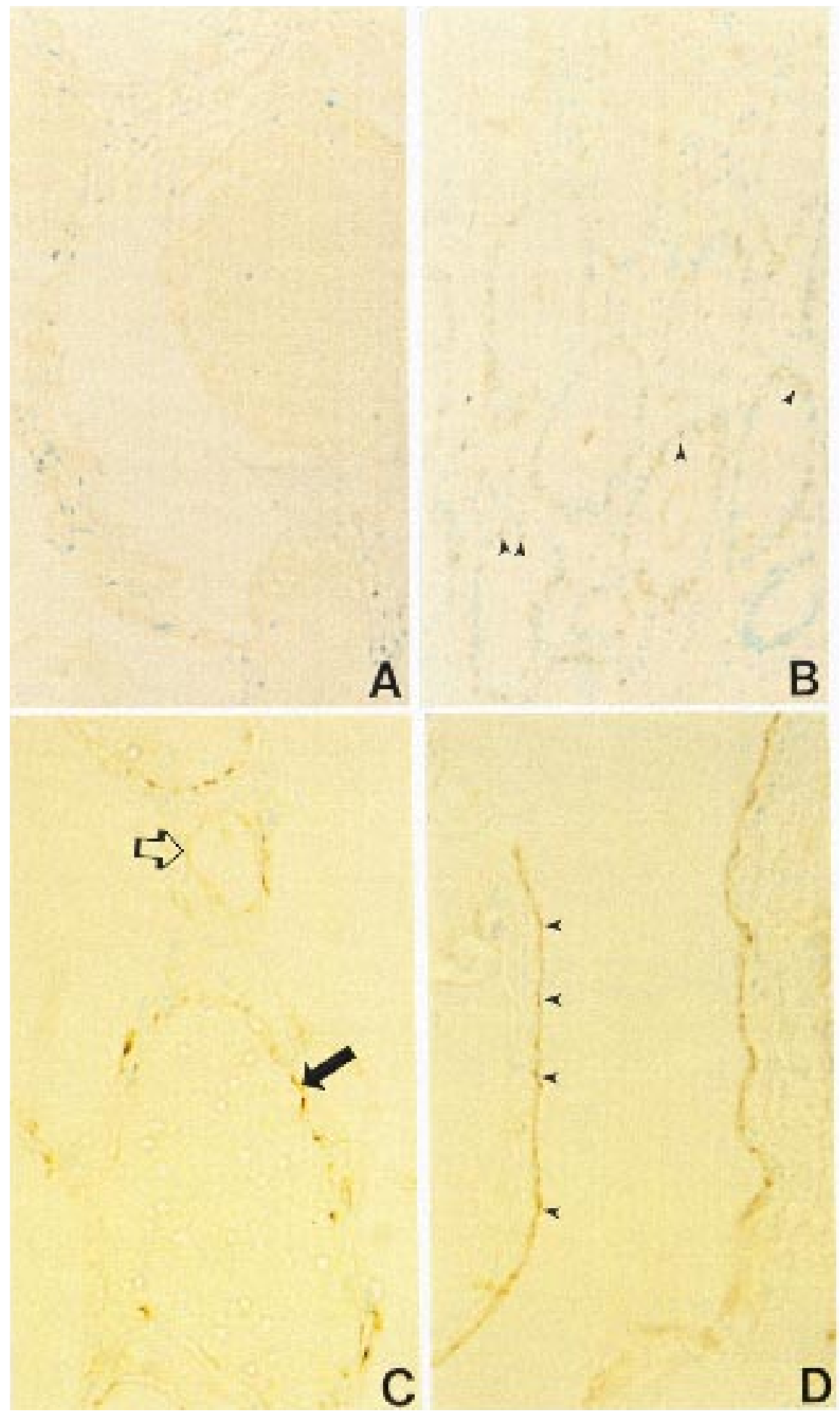

Fig 4. Immunohistochemical staining for PCNA for patients with pulmonary AV malformations (A) and positive control (intestinal epithelium, B). Note frequent nuclear staining in the positive control (arrowheads )that is absent in pulmonary AV malformation tissue (methyl green counterstain, 450×). Immunohistochemical staining for CD31 demonstrating minimal (solid ar row) or no (hollow ar row) staining for pulmonary AV malformations vessels (C) compared with dense staining of endothelium in blood vessels of controls (arrowheads )(D) (methyl green counterstain, 450×). 


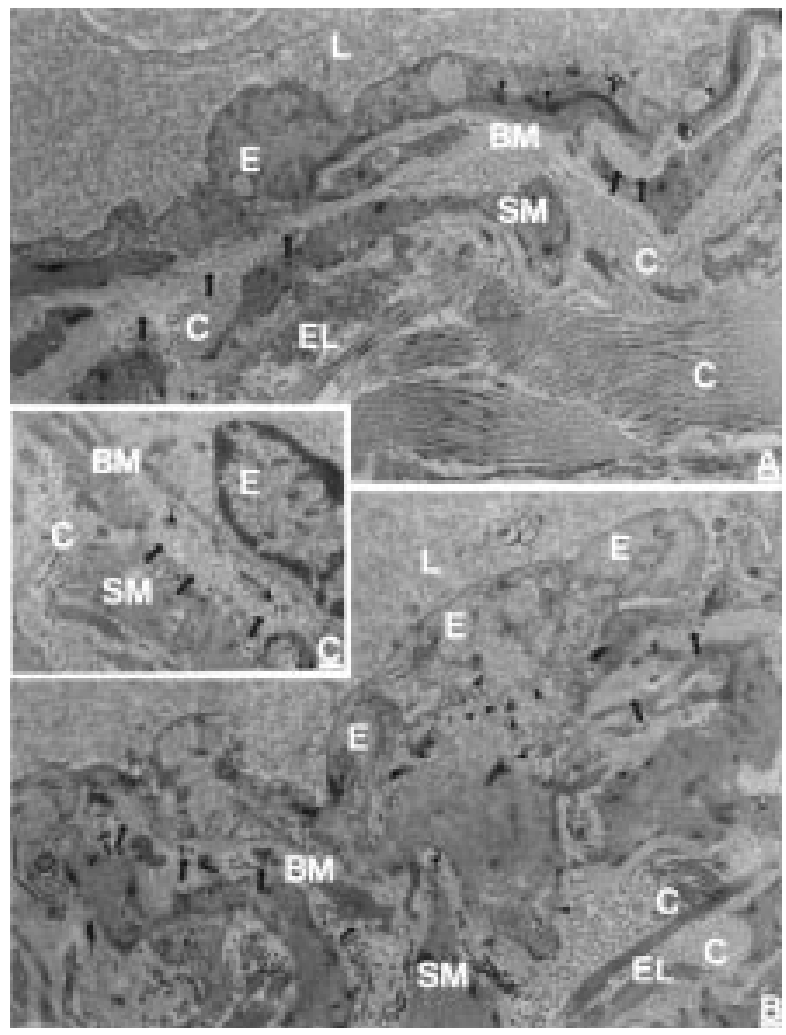

Fig 5. Electron micrographs of lung biopsy tissue from patient with pulmonary AV malformations. A, Wall of pulmonary AV malformations demonstrates the lumen $(L)$ and an endothelial cell $(E)$ attached to an abnormal basement membrane $(B M)$. The basement membrane appears to be irregular, thickened, and multilayered in places (arrows). The endothelial cell contains stress fiber bundles (arrowheads). Also demonstrated are elastin fibers $(E L)$, collagen fibers $(C)$, and smooth muscle $(S M)$. B, Abnormal appearance of endothelium $(E)$ of pulmonary AV malformation endothelium, demonstrating thickening and irregularity (arrowheads). Collagen fibers (arrows) are demonstrated in the thickened basement membrane $(B M)$. Smooth muscle $(S M)$, elastin fibers $(E L)$, and collagen bundles $(C)$ are also seen beneath the basement membrane. L, Lumen. $\mathbf{C}$, An area of basement membrane $(B M)$ thickening (arrows) demonstrating many collagen fibrils (arrowheads) loosely attached to the endothelium $(E)$. $S M$, Smooth muscle; $C$, collagen fibers below basement membrane.

diminished CD31 staining and may be due to decreased intercellular junctions between the endothelial cells of the pulmonary AV malformations.

\section{Discussion}

Previous reports have identified patients at risk for the development of pulmonary AV malformations after pal- liative operations for congenital heart disease. ${ }^{1-3}$ All of these palliative procedures share in common the provision of pulmonary arterial blood flow derived from systemic venous blood devoid of hepatic drainage. Further evidence for the role of the liver in the formation of these lesions comes from the observation that clinically similar pulmonary AV malformations occur in patients with advanced hepatic failure and are curable by liver transplantation. ${ }^{6}$ On the basis of these observations, a viable hypothesis is that pulmonary AV malformations develop in the lungs of these patients because of the absence of a hepatic factor in the pulmonary arterial circulation. On the basis of this premise, we have demonstrated an inhibitory effect of the conditioned media derived from cultured hepatocytes on endothelial proliferation and have partially purified this activity. ${ }^{4}$

The present study attempts to provide a detailed analysis of the histologic and ultrastructural features of pulmonary AV malformations that form in children with some forms of cyanotic congenital heart disease. We were successful in identifying the microscopic lesion associated with the physiologic abnormality of pulmonary AV malformations, finding greatly increased numbers of vessels with 2 different morphologic types-lakes of dilated, thin-walled vessels and chains of clustered, smaller vessels. This was a diffuse process extending into regions of the lung periphery that did not appear to be significantly involved on preoperative angiography. The demonstration of branches from the precapillary arterioles into these thin-walled vessels may represent the histologic correlate of the right-to-left shunt that these children demonstrate. Blood leaving the precapillary arterioles at this level and returning to the heart would presumably remain desaturated and not be available for gas exchange. The determination of microvessel density, which has proven to have prognostic significance in tumor specimens, ${ }^{7}$ may have some clinical utility in grading the severity of pulmonary AV malformations in lung biopsy tissue. However, the microvessel density results have broader implications in terms of the angiogenic potential of this tissue. In oncologic studies, increased microvessel density is usually associated with accelerated angiogenesis resulting in an increased proliferative state of the tumor. These results suggest that pulmonary AV malformations that develop after cavopulmonary anastomosis might be similar to other conditions that are due to abnormally increased angiogenesis.

Pulmonary AV malformations in these patients, however, show no evidence of excess proliferative activity by immunohistologic techniques. The specific staining patterns for endothelial and proliferation markers were 
unique when compared with patterns in other lesions that demonstrate intense vascular proliferation, such as soft tissue hemangiomas. ${ }^{8}$ Proliferating phase hemangiomas are angiogenically active neoplasms that are composed of vast numbers of small blood vessels with a high proliferative activity. Proliferating hemangiomas are PCNA positive and demonstrate positive staining for $\mathrm{CD} 31$. The pulmonary AV malformations were PCNA negative and stained only faintly for CD31 in a minority of the vessels, with many of the vessels demonstrating no CD31 staining. PCNA is a nuclear protein that is associated with the control of cell cycle progression and is expressed in tissues that are rapidly proliferating. ${ }^{9}$ Tissues that demonstrate active angiogenesis usually contain endothelial cells that stain positively for PCNA. These results suggest that vascular expansion has occurred in pulmonary AV malformations without the exuberant cellular proliferation that might be expected. A possible explanation is that endothelial cell spreading and migration may be responsible for "new" blood vessel formation in these lesions. Alternatively, the absence of excessive cellular proliferation may signify that pulmonary AV malformations arise from the distention and dilatation of previously existing vascular channels that enlarge as collateral vessels in response to pressure gradients between the SVC and the IVC (after a Glenn procedure) or the mesenteric venous circulation (after a Kawashima procedure).

CD31 is a platelet and endothelial adhesion molecule that is present at intercellular junctions between endothelial cells. ${ }^{10}$ The diminished CD31 staining in the pulmonary AV malformations is consistent with the discontinuity of the endothelium visualized by electron microscopic studies; alternatively, it may reflect downregulation of this endothelial surface marker. Decreased intercellular junctions between the endothelial cells might have resulted in the edema that could be visualized histologically (Fig 2, A) and may have etiologic implications regarding capillary leak and pleural effusions, which these children often have after surgery. Another interesting observation from the electron microscopic results was the demonstration of disordered collagen fibrils and disruption of the basement membrane, as well as endothelial irregularity. Another possible explanation for these electron microscopic changes could be due to a component of injury response with collagen disruption and prominent interstitial edema. The source of this injury is not clear but could be a host response to a number of physiologic alterations that these children experience, such as chronic hypoxia or nonpulsatile pulmonary blood flow.
The rarity of this condition is reflected in the fact that only 2 patients have undergone this in-depth histologic analysis. As these techniques are applied to larger numbers of patients, their more general applicability for this lesion can be determined. The role of blood vessel proliferation versus vascular dilatation has been raised as an area of question that has not been definitively established by this work. Finally, the role of the liver in the development of these lesions has not been addressed by these techniques. Ideally, however, this work will serve as a preliminary inquiry that will lead to future studies that further our understanding of the nature and etiology of this condition.

\section{Summary}

The present study is an attempt to establish the histologic and ultrastructural features of pulmonary AV malformations that form in the lungs of children with certain cyanotic congenital heart lesions associated with pulmonary blood flow that is devoid of hepatic venous effluent. Microvessel density was determined and immunohistochemical staining for markers of angiogenesis was undertaken to determine the angiogenic potential of these lesions. The microvessel density results suggest that this tissue is angiogenically active. However, the immunohistochemical results suggest that greatly increased endothelial proliferation is not occurring. Future studies should be aimed at distinguishing the effects of cellular proliferation from those of vascular dilatation and cell spreading in the etiology of these lesions. The possible role of the liver in the formation of pulmonary AV malformations is not further elucidated by these studies; ideally, however, this report will provide a basis for future detailed analyses of patients with this condition.

\section{REFERENCES}

1. McFaul RC, Tajik AJ, Mair DD, Danielson GK, Seward JB. Development of pulmonary arteriovenous shunt after superior vena cava-right pulmonary artery (Glenn) anastomosis. Circulation 1977;55:212-6.

2. Srivastava D, Preminger T, Lock JE, Mandell V, Keane JF, Mayer $\mathrm{JE}$, et al. Hepatic venous blood and the development of pulmonary arteriovenous malformations in congenital heart disease. Circulation 1995;92:1217-22.

3. Bernstein H, Brook MM, Silverman NH, Bristow J. Development of pulmonary arteriovenous fistulae in children after cavopulmonary shunt. Circulation 1995;92(Suppl):II309-14.

4. Marshall B, Duncan BW, Jonas RA. A possible role of angiogenesis in the development of pulmonary arteriovenous malformations in children after cavopulmonary anastomosis. Cardiol Young 1997;7:370-4.

5. Lupinetti FM, Kneebone JM, Rekhter MD, Brockbank KG, Gordon D. Procollagen production in fresh and cryopreserved aortic valve grafts. J Thorac Cardiovasc Surg 1997;113:102-7. 
6. Stoller JK, Moodie D, Schiavone WA, Vogt D, Broughan T, Winkelman E, et al. Reduction of intrapulmonary shunt and resolution of digital clubbing associated with primary biliary cirrhosis after liver transplantation. Hepatology 1990;11:54-8.

7. Leon SP, Folkerth RD, Black PM. Microvessel density is a prognostic indicator for patients with astroglial brain tumors. Cancer 1996;77:362-71.
8. Takahashi K, Mulliken JB, Kozakewich HPW, Rogers RA, Folkman J, Ezekowitz RAB. Cellular markers that distinguish the phases of hemangioma during infancy and childhood. J Clin Invest 1994;93:2357-64.

9. Kelman Z. PCNA: structure, functions and interactions. Oncogene 1997; 14:629-40.

10. Newman PJ. The biology of PECAM-1. J Clin Invest 1997;99:3-8. 